\title{
Effects of Poly(ethylene glycol) (PEG) Concentration on the Permeability of PEG-Grafted Liposomes
}

\author{
Kaname Hashizaki, ${ }^{*, a}$ Hiroyuki TAgUchi, ${ }^{a}$ Chika ItoH, ${ }^{b}$ Hideki SAKaI,${ }^{c}$ Masahiko Abe,${ }^{c}$ \\ Yoshihiro SAITO, ${ }^{a}$ and Naotake OGAWA ${ }^{a}$ \\ ${ }^{a}$ College of Pharmacy, Nihon University; 7-7-1 Narashinodai, Funabashi, Chiba 274-8555, Japan: ${ }^{b}$ DDS Development \\ Division, NOF Co.; 3-3 Chidori-cho, Kawasaki, Kanagawa 210-0865, Japan: and ${ }^{c}$ Faculty of Science and Technology, \\ Tokyo University of Science; 2641 Yamazaki, Noda, Chiba 278-8510, Japan. \\ Received May 31, 2004; accepted October 28, 2004
}

Poly(ethylene glycol)-grafted liposomes (PEG-liposomes) were prepared from dipalmitoylphosphatidylcholine (DPPC) with various amounts of distearoyl- $N$-monomethoxy poly(ethylene glycol)-succinyl-phosphatidylethanolamines (DSPE-PEG) with PEG molecular weights of 1000, 2000, 3000 and 5000. The effects of DSPE-PEG concentration on the permeability of PEG-liposomes were investigated using carboxyfluorescein (CF). In the gel state, the CF leakage from PEG-liposomes was decreased with increasing mole fractions of DSPE-PEG for all PEG molecular weights. In the liquid-crystalline state, the CF leakage from PEG-liposomes containing DSPE-PEG1000 gradually increased with increasing mole fractions of DSPE-PEG, while that of PEG-liposomes whose molecular weight in PEG units was above 2000 rapidly decreased by the addition of DSPE-PEG. Furthermore, no effect of PEG molecular weight on CF leakage was observed. The relationship between the fluorescence polarization of 1,6-diphenyl-1,3,5-hexatriene (DPH) (or 1-(4-trimethylammoniumphenyl)6-phenyl-1,3,5-hexatriene (TMA-DPH)) and the mole fraction of DSPE-PEG for PEG-liposomes was also investigated. No significant changes in fluorescence polarization of DPH for liposomal bilayer membranes was observed in the gel and liquid-crystalline states due to the addition of DSPE-PEG, while that of TMA-DPH was decreased compared with that of liposomes without DSPE-PEG in both states.

Key words liposome; poly(ethylene glycol); permeability; fluorescence polarization; carboxyfluorescein

Liposomal drug delivery systems (DDS) have been widely researched for use in drug toxicity reduction and/or drug targeting to desired target tissues. ${ }^{1)}$ However, liposomes are rapidly removed from the circulation following their intravenous administration primarily by Kupffer cells in the liver and fixed macrophages in the spleen. Thus, it is important to develop modified liposomes that are able to avoid uptake by the reticuloendothelial system (RES) and extend their circulation half-life in vivo. Many studies have reported that the conjugation of amphipathic polyethylene glycol (PEG) with liposomes (PEG-liposomes) significantly increases the blood circulation liposome half-life compared with those without PEG. $^{2-5)}$ In our previous studies, ${ }^{6,7)}$ we reported on the effect of PEG-lipid PEG chain length on the membrane characteristics of liposomes. The addition of PEG-lipid to liposomes caused lateral phase separation in the gel and liquid-crystalline states, and the fluidity in the interfacial region of liposomal bilayer membranes was markedly increased by the addition of PEG-lipids. In addition, the permeability of liposomal bilayer membranes decreased compared with those without PEG. From these results, we concluded that the solute permeability of PEG-liposomes is affected by not only the properties of the liposomal bilayer membranes such as phase separation and membrane fluidity, but also the PEG chain length of the liposomal surface.

In this study, we investigated the effects of PEG-lipid concentration on CF leakage from PEG-liposomes to elucidate the permeation mechanism of PEG-liposomes in more detail.

\section{Experimental}

Materials $\quad \mathrm{L}-\alpha$-Dipalmitoylphosphatidylcholine (DPPC，99.6\% pure) was obtained from NOF Co., Ltd., and Distearoyl- $N$-monomethoxy poly(ethylene glycol)-succinyl-phosphatidylethanolamines (DSPE-PEG) were acquired from NOF Co., Ltd. (Tokyo, Japan). The weight-average molecular weights of poly(ethylene glycol) were 1000, 2000, 3000 and 5000. 5-(6)Carboxyfluorescein (CF, 99\% pure) was purchased from Molecular Probes, Inc. (OR, U.S.A.) and was used without further purification. 1,6-Diphenyl1,3,5-hexatriene (DPH, 98\% pure) and 1-(4-trimethylammoniumphenyl)-6phenyl-1,3,5-hexatriene (TMA-DPH, 95\% pure) were purchased from Sigma Chemical Co. (MO, U.S.A.) and were also used without further purification. Dulbecco's Phosphate-buffered saline (PBS) powder composed of $\mathrm{NaCl}, \mathrm{KCl}, \mathrm{Na}_{2} \mathrm{HPO}_{4}$ and $\mathrm{KH}_{2} \mathrm{PO}_{4}$ was purchased from Wako Pure Chemical Industries, Ltd. (Osaka, Japan). PBS powder was dissolved in water for injection (Otsuka Pharmaceutical Co., Ltd., Tokyo, Japan), and an isotonic solution of $\mathrm{pH} 7.4$ was used. All other chemicals were commercial products of reagent grade.

Preparation of PEG-Liposomes PEG-liposomes were prepared using DPPC and DSPE-PEG, which were first dissolved in chloroform in a test tube. The solvent was then removed by blowing nitrogen gas into the test tube, and the residual solvent was further dried overnight at room temperature in a desiccator under a vacuum. PBS was added to the lipid film and warmed $\left(55-60^{\circ} \mathrm{C}\right)$ above the phase transition temperature of DPPC $\left(41^{\circ} \mathrm{C}\right)$ for $30 \mathrm{~min}$. The test tube was then vigorously shaken in a vortex mixer, and multilamellar vesicles (MLV) were obtained. Large unilamellar vesicles (LUV) were obtained from MLV suspensions extruded five times through a double-stacked polycarbonate membrane filter (pore size: $200 \mathrm{~nm}$ ) using an Extruder (Lipex Biomembranes Inc., British Columbia, Canada) at $55^{\circ} \mathrm{C}$.

Permeability Measurements To estimate solute leakage from the liposomes, $\mathrm{CF}$ as a solute marker was encapsulated, and $\mathrm{CF}$ leakage was measured using a dialysis technique similar to that of Johnson et al. and Nikolova et al. ${ }^{8,9}$ Briefly, liposomes were prepared as described above, except that $50 \mathrm{~mm}$ CF was included. Liposomes encapsulating CF were separated from unencapsulated CF by gel chromatography on a Sephadex G-50 column (Amersham Pharmacia Biotech, Uppsala, Sweden) with PBS as the eluant. A liposome solution $(1 \mathrm{ml})$ was placed in a dialysis bag (Spectra/Por Membrane No. 7, Spectrum Laboratories Inc., CA, U.S.A.), and it was dialyzed at $4{ }^{\circ} \mathrm{C}$ to remove unencapsulated CF. Beforehand, $100 \mathrm{ml}$ of PBS used for dialysis fluid was placed in a screw-cap bottle and kept at the desired temperature. The dialysis bag enclosing the liposome solution was transferred to the screw-cap bottle, and the dialysis fluid was stirred with a magnetic stirrer. Aliquots of the fluid were withdrawn at appropriate time intervals, and the fluorescence intensity was then measured with a spectrofluorometer (FP-777, JASCO Co., Tokyo, Japan). The excitation and emission 
wavelengths were $490 \mathrm{~nm}$ and $520 \mathrm{~nm}$, respectively. The samples were returned to the bottle after each measurement to avoid changes in the dialysis fluid volume. After the measurements were completed, the dialysis bag was cut open in the bottle, and the total CF fluorescence intensity was determined after the addition of $10 \%$ Triton $\mathrm{X}-100$ solution.

Fluorescence Polarization Measurements The fluidity of the liposomal bilayer membranes was determined from fluorescence polarization $(P)$ measurements using a fluorescent probe. DPH and TMA-DPH were dissolved in tetrahydrofuran and ethanol, ${ }^{10)}$ respectively. The fluorescent probe was added to the dilute liposome solution at a molar ratio of lipid to probe of $300: 1$ (DPH) or $600: 1$ (TMA-DPH) and incubated for $1 \mathrm{~h}$ at $55^{\circ} \mathrm{C}$. Fluorescence polarization $(P)$ was calculated according to the following equation:

$$
P=\left(I_{\mathrm{P}}-G I_{\mathrm{V}}\right) /\left(I_{\mathrm{P}}+G I_{\mathrm{v}}\right)
$$

where $I_{\mathrm{P}}$ and $I_{\mathrm{V}}$ are the fluorescence intensities of the emitted light polarized parallel and vertical to the excitation light, respectively, and $G$ is the grating correction factor. ${ }^{11)} I_{\mathrm{P}}$ and $I_{\mathrm{V}}$ were measured with a spectrofluorometer. DPH was excited at $350 \mathrm{~nm}$ and fluorescence emission was detected at $450 \mathrm{~nm}$. When TMA-DPH was used, the excitation and emission wavelengths were $360 \mathrm{~nm}$ and $430 \mathrm{~nm}$, respectively.

\section{Results and Discussion}

CF Leakage from Liposomes The effects of a PEGlipid on the encapsulation efficiency of liposomes was examined. Figure 1 shows the relationship between the relative encapsulation efficiency of CF in liposomes and the mole fraction of DSPE-PEG. The relative encapsulation efficiency indicates the ratio of the encapsulation efficiency of PEG-lipo- somes to that of liposomes without DSPE-PEG. The relative encapsulation efficiency of PEG-liposomes containing DSPE-PEG1000 was not significantly changed by the addition of DSPE-PEG. On the other hand, the relative encapsulation efficiency of PEG-liposomes whose molecular weight in PEG units was above 2000 was increased by the minute addition of DSPE-PEG, and the relative encapsulation efficiency significantly decreased at mole fractions above $0.090-0.117$. The reason for this is that the liposomes coexist with the mixed micelles at a high PEG-lipid concentration. It is also reported that the fraction of the PEG-lipid incorporated into liposomal bilayer membranes decreased with increasing PEG-lipid concentrations and molecular weight in PEG units. ${ }^{12,13)}$ Furthermore, Sriwongsitanont et al. ${ }^{14)}$ examined the effects of PEG-lipid concentration on liposomal structure. They reported that liposomes coexisted with mixed micelles when the concentration of DSPE-PEG2000 was greater than $10 \mathrm{~mol} \%$. This is in good agreement with our results.

The effects of PEG-lipid concentration on CF leakage from liposomes was examined. In general, when the solute leakage from the liposomes is discussed, differences in the liposomal bilayer thickness and particle sizes should be taken into consideration. ${ }^{15)}$ In our previous study, ${ }^{7)}$ we confirmed that the addition of DSPE-PEG to the DPPC liposomal bi-
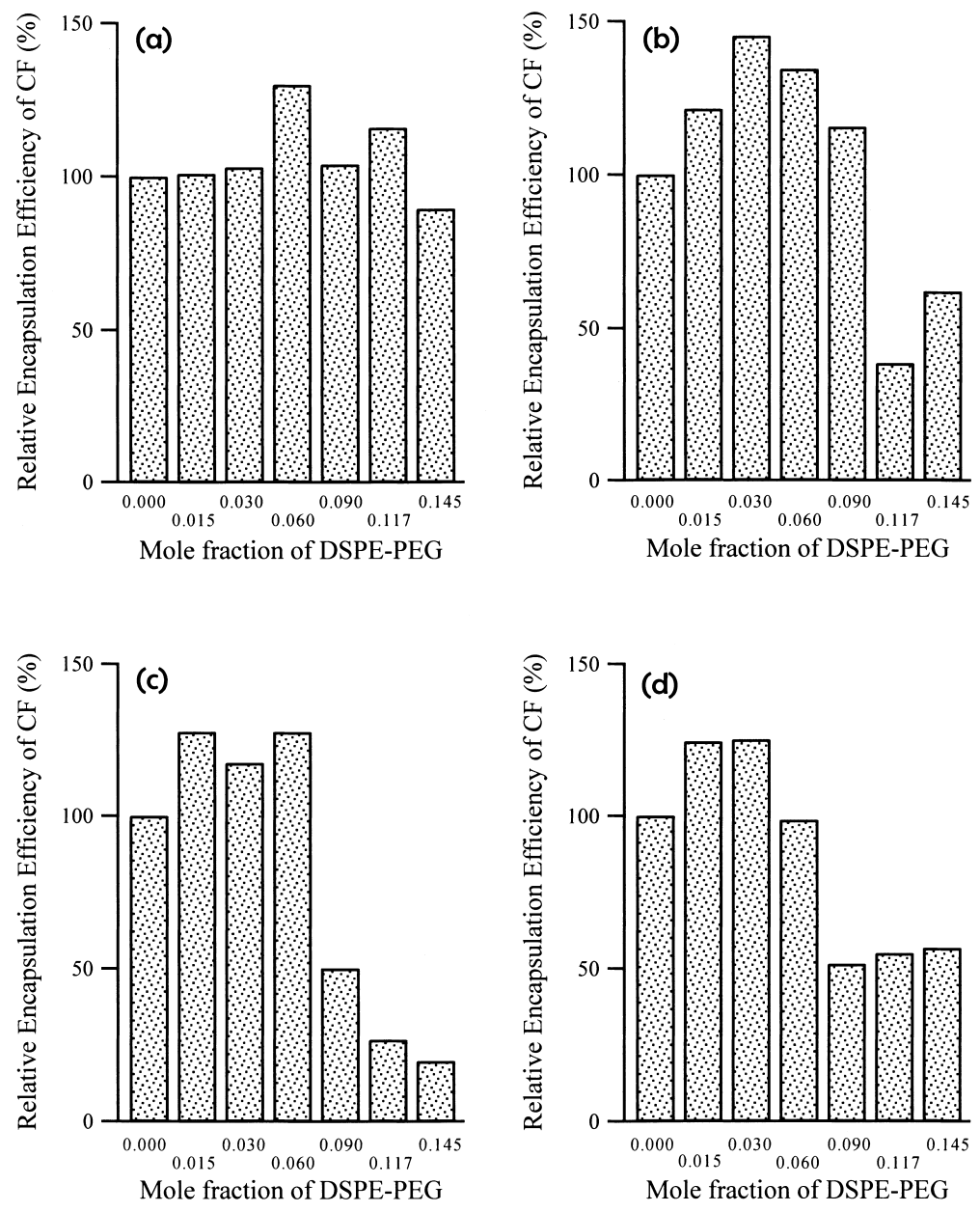

Fig. 1. The Relationship between Relative Encapsulation Efficiency of CF in Liposomes and the Mole Fraction of DSPE-PEG1000 (a), DSPE-PEG2000 (b), DSPE-PEG3000 (c) and DSPE-PEG5000 (d) 
layer membrane caused lateral phase separation, and it seems that the thickness of liposomal bilayer membranes is not uniform. Furthermore, it is necessary to calculate the liposomal bilayer thickness for PEG-liposomes considering the thickness of the PEG chain layer on the liposomal surface. However, it is very difficult to obtain the thickness of the PEG chain layer because the conformation of the PEG chain is changed with increasing concentrations of PEG-lipid. ${ }^{16,17)}$ Therefore, in the present study, CF leakage is discussed in terms of the rate constant. The rate constant of CF leakage was calculated according to the following equation:

$$
\frac{F I_{\mathrm{o}} \cdot v_{0}}{F I_{\mathrm{i}} \cdot v_{2}}=1-\frac{1}{K-L}\left\{(1-\alpha) K e^{-L t}-(L-\alpha K) e^{-K t}\right\}
$$

where $F I_{\mathrm{o}}$ and $F I_{\mathrm{i}}$ are the fluorescence intensities outside and inside the dialysis bag, respectively, $v_{0}$ is the total volume of the system, $v_{2}$ is the external solution (dialysis fluid) volume, $K$ and $L$ are rate constants of CF leakage from the dialysis bag and liposome, respectively, and $\alpha$ is the fraction of CF that leaks out of the dialysis bag from the liposomes before the start of the experiment. $K$ in Eq. 2 can be determined according to the following equation using a $\mathrm{CF}$ solution instead of a liposome solution.

$$
\frac{F I_{\mathrm{o}} \cdot v_{0}}{F I_{\mathrm{i}} \cdot v_{2}}=1-e^{-K t}
$$

Figure 2 shows plots according to Eq. 2 for CF leakage from liposomes together with calculated curves that best fit the experimental data. The resulting $L$-values are plotted as a function of DSPE-PEG concentration, as shown in Fig. 3.

In our previous study, ${ }^{6}$ ) we reported that all PEG-liposomes caused gel-liquid crystalline phase transition at $41-$ $43^{\circ} \mathrm{C}$, as seen by differential scanning calorimetry (DSC) measurements. Therefore, the liposomal bilayer membrane at 30 and $37^{\circ} \mathrm{C}$ is in the gel state, and at $50^{\circ} \mathrm{C}$ is in the liquidcrystalline state, respectively. In the gel state, the $L$-value of PEG-liposomes containing DSPE-PEG1000 gradually decreased with increasing mole fractions of DSPE-PEG, while the $L$-value for the liquid-crystalline state increased with increasing mole fractions of DSPE-PEG, as shown in Fig. 3.

On the other hand, the $L$-value of PEG-liposomes whose molecular weight in PEG units was above 2000 rapidly de- creased following the minute addition of DSPE-PEG in the gel and liquid-crystalline states. Furthermore, no effect of the PEG molecular weight of the PEG-lipid on the $L$-value was observed.

Fluorescence Polarization of Liposomal Bilayer Membranes The fluidity of liposomal bilayer membranes was investigated using the fluorescent probes DPH and TMA$\mathrm{DPH}$. The fluorescence polarization of DPH indicates the fluidity in the hydrocarbon region of a liposomal bilayer membrane, and that of TMA-DPH indicates the hydrophobic-hydrophilic interfacial region. ${ }^{10)}$
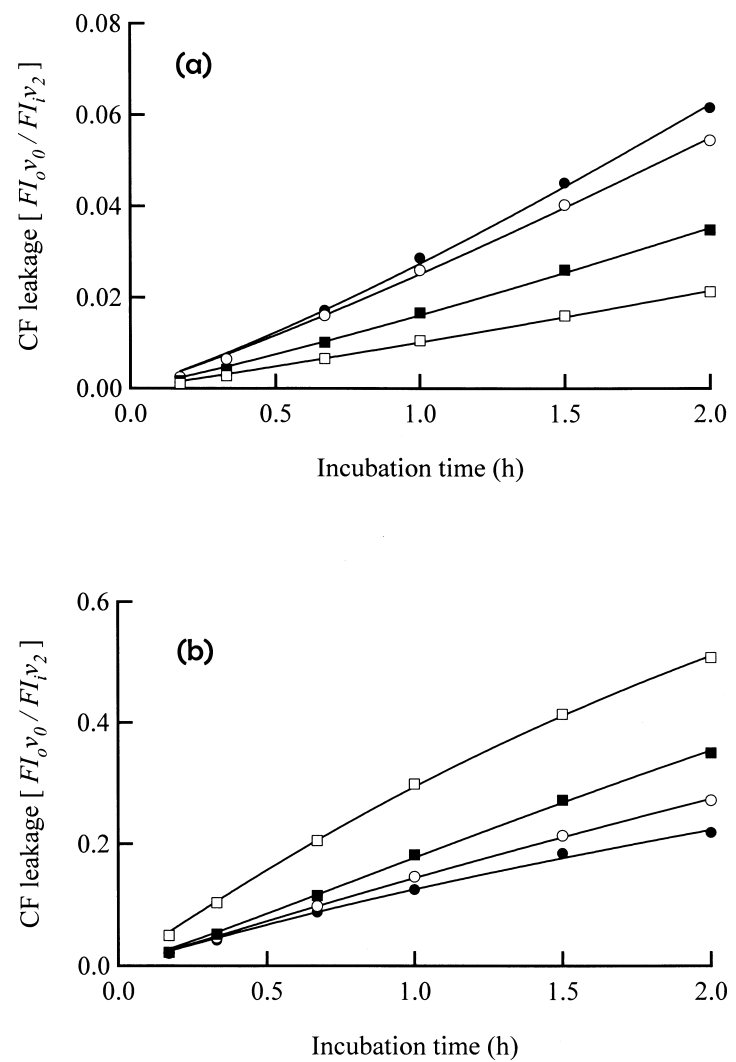

Fig. 2. Best-Fit Curves for CF Leakage from PEG-Liposomes Containing DSPE-PEG1000 Based on Eq. 2 at $37^{\circ} \mathrm{C}$ (a) and $50{ }^{\circ} \mathrm{C}$ (b)

The mole fractions of DSPE-PEG were $\bullet, 0 ; \bigcirc, 0.030 ; \mathbf{\square}, 0.090 ; \square, 0.145$.
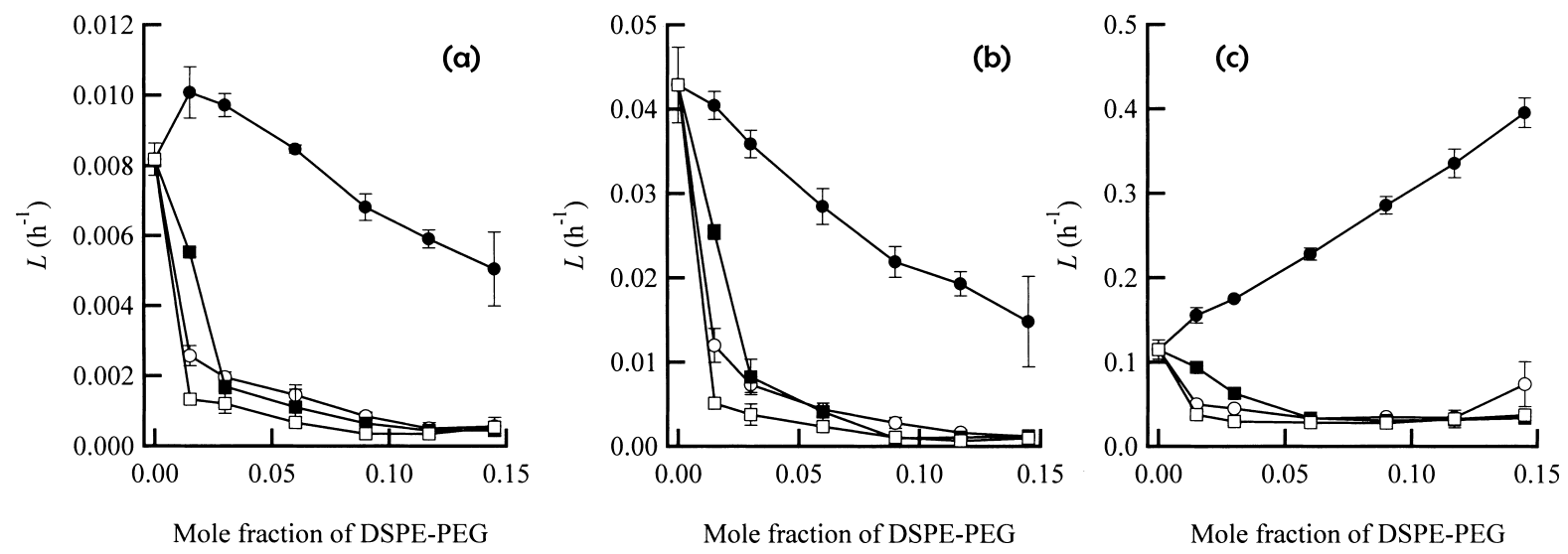

Fig. 3. The Relationship between the Rate Constant $(L)$ for CF Leakage from PEG-Liposomes and the Mole Fraction of DSPE-PEG at $30^{\circ} \mathrm{C}(\mathrm{a}), 37^{\circ} \mathrm{C}(\mathrm{b})$ and $50^{\circ} \mathrm{C}(\mathrm{c})$

-: DPPC/DSPE-PEG1000, O: DPPC/DSPE-PEG2000, 5 experiments. 

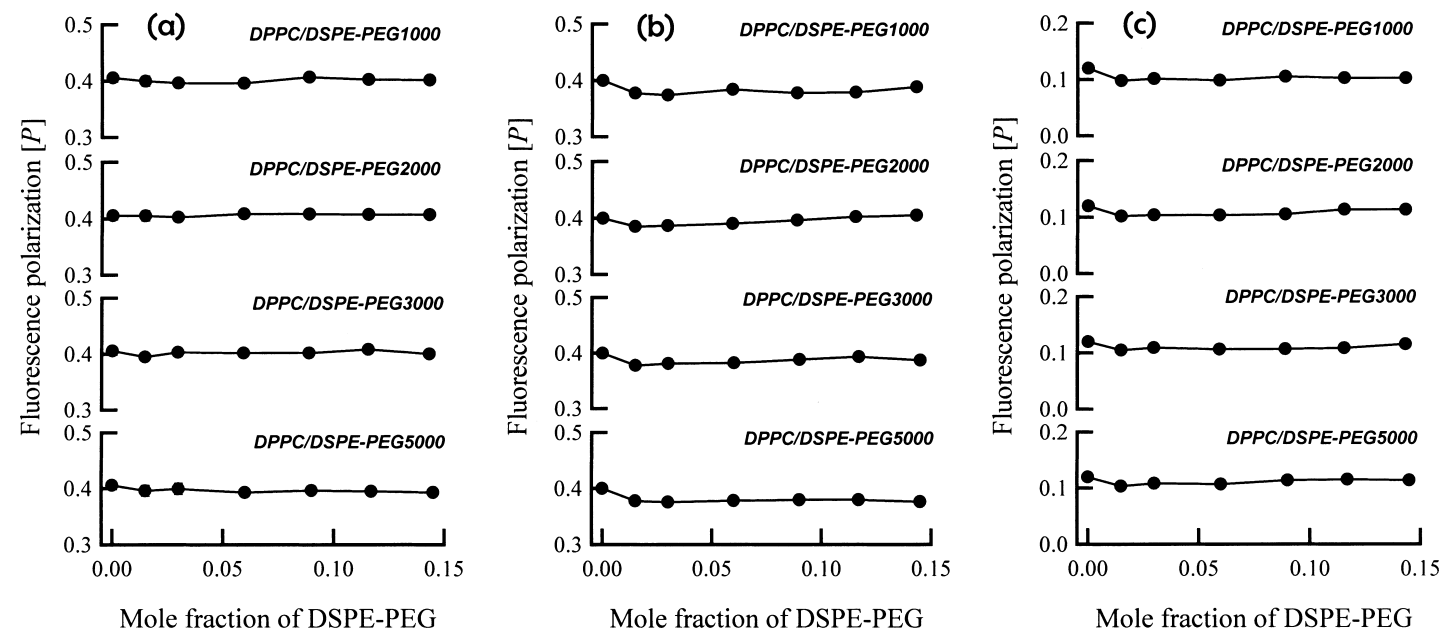

Fig. 4. The Relationship between Fluorescence Polarization of DPH and the Mole Fraction of DSPE-PEG for PEG-Liposomes at $30^{\circ} \mathrm{C}(\mathrm{a}), 37^{\circ} \mathrm{C}(\mathrm{b})$ and $50^{\circ} \mathrm{C}(\mathrm{c})$

Each point with a vertical bar represents the mean \pm S.E. of 3 experiments.
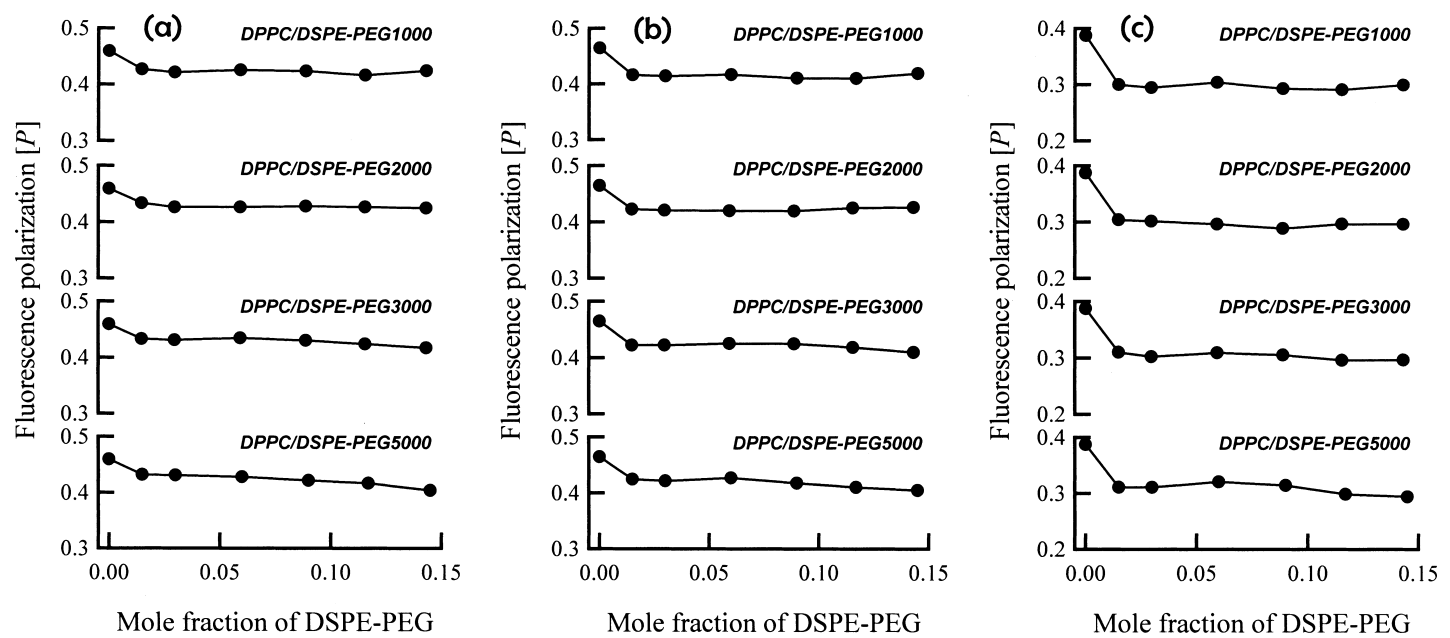

Fig. 5. The Relationship between Fluorescence Polarization of TMA-DPH and the Mole Fraction of DSPE-PEG for PEG-Liposomes at $30{ }^{\circ} \mathrm{C}(\mathrm{a}), 37^{\circ} \mathrm{C}$ (b) and $50^{\circ} \mathrm{C}(\mathrm{c})$

Each point with a vertical bar represents the mean \pm S.E. of 3 experiments.

The relationship between the fluorescence polarization of DPH and the mole fraction of DSPE-PEG for PEG-liposomes is shown in Fig. 4. No significant changes in the fluorescence polarization of DPH for liposomal bilayer membranes were found for either state due to the addition of DSPE-PEG. Thus, the addition of DSPE-PEG did not affect fluidity in the hydrocarbon region of the liposomal bilayer membrane.

Next, the relationship between the fluorescence polarization of TMA-DPH and the mole fraction of DSPE-PEG for PEG-liposomes was investigated, and the results are shown in Fig. 5. The fluorescence polarization of TMA-DPH for PEG-liposomes was decreased compared with that of liposomes without DSPE-PEG in both states. Therefore, the fluidity in the interfacial region of the liposomal bilayer membrane increased due to the addition of DSPE-PEG. This phenomenon originates from a weakening of the interaction near the hydrophilic group of DPPC molecules due to the bulky PEG chain of the PEG-lipid. In addition, the fluorescence polarization of TMA-DPH for PEG-liposomes hardly changed, even though the mole fraction and PEG chain length of DSPE-PEG increased. From this, we thus confirmed that morphological changes in the liposomes did not occur using electron microscopy observations (data not shown). If morphological changes had occurred in the range of the DSPEPEG concentration used, fluorescence polarization may have rapidly decreased.

Permeation Mechanisms In spite of the increased fluidity in the hydrophobic-hydrophilic interfacial region of the liposomal bilayer membrane, the CF leakage from PEG-liposomes was suppressed in both states by the addition of DSPE-PEG, except for DSPE-PEG1000 at $50{ }^{\circ} \mathrm{C}$, as shown in Fig. 3. This shows that the fluidity of the liposomal bilayer membrane is not the main factor in CF leakage from PEG-liposomes which consist of this lipid composition. In our previous paper, ${ }^{7)}$ we confirmed that the addition of DSPE-PEG to the DPPC liposomal bilayer membrane causes lateral phase separation in the gel and liquid-crystalline states, as seen by DSC measurements and freeze-fracture electron microscopy observations. Solute leakage from liposomes 
rapidly accelerates when the components which constitute the liposomes cause phase separation. ${ }^{15,18)}$ However, CF leakage from liposomes in the gel state decreased with increasing mole fractions of DSPE-PEG, and was suppressed even for all PEG molecular weights. Therefore, in the gel state, the effect of phase separation on CF leakage can be decreased by decreasing the mobility of lipid molecules which constitute the liposomal bilayer membrane. In addition, the PEG chains of DSPE-PEG are exposed from the liposomal surfaces when DSPE-PEG forms liposomes with DPPC. ${ }^{3)}$ The exposed PEG chains shield the CF leakage from the liposomes, and the shielding effect of DSPE-PEG increases with increasing mole fractions of DSPE-PEG. On the other hand, the effect of phase separation on CF leakage increases because the mobility of the lipid molecule increases in the liquid-crystalline state. CF leakage from liposomes containing DSPEPEG1000 increased, since the accelerating effect due to the phase separation increased more than the shielding effect due to the PEG chain with increasing mole fractions of DSPEPEG. The shielding effect due to the PEG chain is superior to the accelerating effect caused by the phase separation, which explains why CF leakage decreased in PEG-liposomes whose molecular weight in PEG units was above 2000. Furthermore, there was hardly any difference in the effect of the PEG molecular weight of the PEG-lipids on CF leakage from PEG-liposomes when PEG-lipids with a molecular weight in PEG units above 2000 were used.

\section{References}

1) Gregoriadis G., "Liposomes as Drug Carriers. Recent Trend and Progress," John Wiley \& Sons, New York, 1988.
2) Klibanov A. L., Maruyama K., Torchilin V. P., Huang L., FEBS Lett., 268, 235-237 (1990).

3) Klibanov A. L., Maruyama K., Beckerleg A. M., Torchilin V. P., Huang L., Biochim. Biophys. Acta, 1062, 142-148 (1991).

4) Allen T. M., Hansen C., Martin F., Redemann C., Yau-Young A., Biochim. Biophys. Acta, 1066, 29-36 (1991).

5) Woodle M. C., Lasic D. D., Biochim. Biophys. Acta, 1113, 171-199 (1992).

6) Hashizaki K., Itoh C., Sakai H., Yokoyama S., Taguchi H., Saito Y., Ogawa N., Abe M., Colloids Surf. B, 17, 275-282 (2000).

7) Hashizaki K., Taguchi H., Itoh C., Sakai H., Abe M., Saito Y., Ogawa N., Chem. Pharm. Bull., 51, 815-820 (2003).

8) Johnson S. M., Bangham A. D., Biochim. Biophys. Acta, 193, 82-91 (1969).

9) Nikolova A. N., Jones M. N., Biochim. Biophys. Acta, 1304, 120-128 (1996).

10) Engelke M., Bojarski P., Bloß R., Diehl H., Biophys. Chem., 90, 157173 (2001).

11) Inoue T., Muraoka Y., Fukushima K., Shimozawa R., Chem. Phys. Lipids, 46, 107-115 (1988).

12) Hashizaki K., Itoh C., Sakai H., Yokoyama S., Taguchi H., Saito Y., Ogawa N., Abe M., J. Jpn. Oil. Chem. Soc., 48, 871—876 (1999).

13) Bedu-Addo F. K., Tang P., Xu Y., Huang L., Pharm. Res., 13, 710717 (1996).

14) Sriwongsitanont S., Ueno M., Chem. Pharm. Bull., 50, 1238-1244 (2002).

15) Nojima S., Sunamoto J., Inoue K., “The Liposomes,” Nankodo, Tokyo, 1988.

16) Kuhl T. L., Leckband D. E., Lasic D. D., Israelachvili J. N., Biophys. J., 66, 1479-1488 (1994)

17) Torchilin V. P., Omelyanenko V. G., Papisov M. I., Bogdanov A. A., Jr., Trubetskoy V. S., Herron J. N., Gentry C. A., Biochim. Biophys. Acta, 1195, 11-20 (1994).

18) Blok M. C., Van der Netu-Kok E. C. M., Van Deenen L. L. M., De Gier J., Biochim. Biophys. Acta, 406, 187-196 (1975). 